\title{
Dynamic Depolarized Light Scattering of Small Round Plasmonic Nanoparticles: When Imperfection is Only Perfect
}

\author{
Sandor Balog, ${ }^{*},, \|$ Laura Rodriguez-Lorenzo, ${ }^{\dagger, \|}$ Christophe A. Monnier, ${ }^{\dagger}$ Benjamin Michen ${ }^{\dagger}$ \\ Marc Obiols-Rabasa, ${ }^{\ddagger}$ Lucia Casal-Dujat, ${ }^{\ddagger}$ Barbara Rothen-Rutishauser, ${ }^{\dagger}$ Alke Petri-Fink, ${ }^{\dagger},{ }^{\dagger}$ \\ and Peter Schurtenberger \\ ${ }^{\dagger}$ Adolphe Merkle Institute, University of Fribourg, 1723 Marly, Switzerland \\ ${ }^{\ddagger}$ Physical Chemistry, Department of Chemistry, Lund University, 22100 Lund, Sweden \\ ${ }^{\S}$ Chemistry Department, University of Fribourg, 1700 Fribourg, Switzerland
}

\begin{abstract}
Although small round gold nanoparticles (Au NPs) possess only a small degree of shape anisotropy, they support localized surface plasmon resonances and exhibit intrinsic optical anisotropy. These inherent features promote depolarized light scattering, whose temporal fluctuations carry information about rotational Brownian dynamics, and thus can be used to describe the size distribution of round Au NPs. We demonstrate that this allows for a much more accurate determination of particle size and polydispersity through depolarized dynamic light scattering when compared to standard particle sizing with light scattering.
\end{abstract}

\section{INTRODUCTION}

Nanoparticles (NPs) are fundamental actors of nanotechnology, and potential applications concerning energy, food, electronics, textiles, and biomedical diagnostics and therapeutics for personalized healthcare, among many others, are actively explored and developed. ${ }^{1}$ In particular, noble metal NPs, such as gold, ${ }^{2}$ have been receiving considerable attention due their unique dielectric properties. The refractive index of gold is composed of a dielectric function with a negative real and a positive imaginary constant. When excited by visible-NIR electromagnetic waves, the NP surface supports coherent and confined oscillations of the conduction-band electrons. This phenomenon is referred to as localized surface plasmon resonance (LSPR). ${ }^{3}$ The LSPR is easily identified in the UV-vis extinction spectrum (Figure 1). Owing to localized surface plasmon resonances, polarized light becomes partially depolarized upon scattering, and enhanced depolarized scattering is attributed to interference effects from excited LSPRs. $^{4}$

In suspension, due to Brownian motion, the scattering intensity exhibits temporal fluctuations, and the statistical properties of the temporal fluctuations carry valuable information, which can be used to describe particle size. ${ }^{5}$ If the NPs are optically anisotropic, then the intensity of the depolarized scattering will also exhibit temporal fluctuations. Indeed, depolarized dynamic light scattering (DDLS) from $\mathrm{Au}$ nanorods, which are evidently anisotropic in morphology, have been now analyzed on numerous occasions in various laboratories. $^{6-10}$ However, to the best of our knowledge,
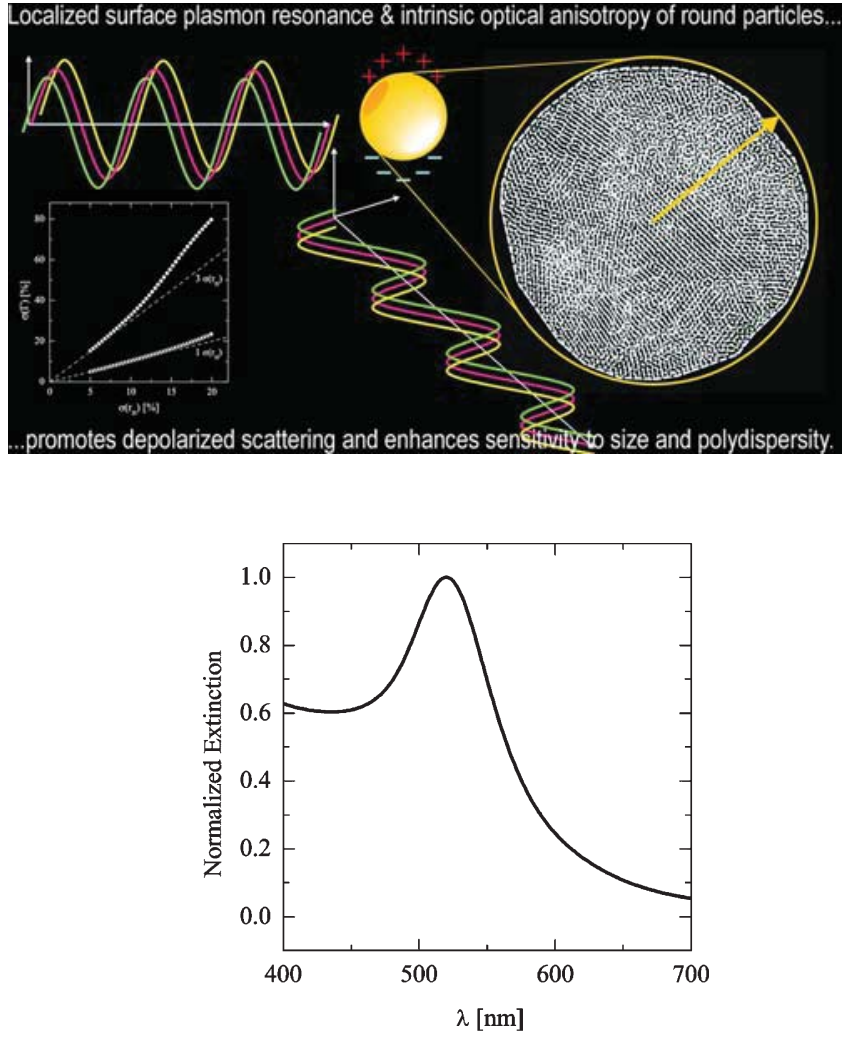

Figure 1. UV-vis extinction spectrum of Au NPs.

DDLS studies addressing round particles with a high degree of spherical symmetry have not been presented.

In this work, it will be shown that despite the lack of pronounced shape anisotropy of small round Au NPs, the presence of intrinsic and inevitable imperfections, existing on a length scale comparable to the particle size, makes DDLS a very sensitive characterization tool for this class of NPs. Owing to the polycrystalline nature, small round Au NPs possess intrinsic and inevitable imperfections, such as grain boundaries and small deviations from the spherical shape. These are strong 
enough to result in optical anisotropy, yet do not restrict the applicability of the straightforward spherical model. This "perfect" balance between these features provides the base of an in situ and practically real-time alternative to the most frequently used techniques: UV-vis spectroscopy ${ }^{11}$ and transmission electron microscopy (TEM).

\section{MATERIALS AND METHODS}

Citrate-stabilized $\mathrm{Au}$ NPs $([\mathrm{Au}]=0.5 \mathrm{mM})$ were synthesized as reported by Turkevich et al. ${ }^{12}$ Aqueous solutions $\left(3.4 \times 10^{-3}\right.$ $\mathrm{mM})$ of thiolated methoxy- $\left(\mathrm{PEG}-\mathrm{CH}_{3}, M_{\mathrm{w}}=5000 \mathrm{~g} / \mathrm{mol}\right)$ poly(ethylene glycol) were sonicated for $15 \mathrm{~min}$, subsequently mixed with $100 \mathrm{~mL}$ of $\mathrm{Au} \mathrm{NP}$ suspension and left to react at 25 ${ }^{\circ} \mathrm{C}$ for $24 \mathrm{~h}$. This mixing ratio is expected to provide approximately 10 molecules for each $\mathrm{nm}^{2}$ of particle surface. The PEGylated particles were centrifuged twice for $1 \mathrm{~h}$ at $10^{4} \mathrm{~g}$ to remove excess polymers, and then redispersed in $10 \mathrm{~mL}$ water. Finally, the particles were transferred to a phosphate buffer (10 $\mathrm{mM}$ sodium phosphate monobasic/disodium phosphate hydrogen, $\mathrm{pH} 7$ ).

$\mathrm{UV}$-vis spectra of the samples were recorded at $25^{\circ} \mathrm{C}$ using a Jasco V-670 spectrophotometer, using $10 \mathrm{~mm}$-path-length quartz cuvettes.

Micrographs of the Au NPs were taken with a Tecnai F20 transmission electron microscope (FEI), operating at $200 \mathrm{kV}$. High-resolution images were recorded with an UltraScanTM 1000 CCD sensor (Gatan, Inc.) with an image resolution of 2048 times 2048 pixels. To prepare the sample, the suspension was spin-coated and dried on carbon-film square mesh copper grids (Electron Microscopy Sciences, CF-300-Cu). The size distribution of the NPs was obtained from these micrographs by using ImageJ particle analysis software (National Institutes of Health NIH, U.S.A.).

Light scattering measurements were performed at constant temperature $\left(21^{\circ} \mathrm{C}\right)$ using a commercial goniometer instrument (3D LS Spectrometer, LS Instruments AG, Switzerland). The primary beam was formed by a linearly polarized and collimated laser beam ( $\mathrm{HeNe}, 632.8 \mathrm{~nm}, 21 \mathrm{~mW})$, and the scattered light was collected by single-mode optical fibers equipped with integrated collimation optics. The collected light was coupled into two high-sensitivity APD detectors (PerkinElmer, Single Photon Counting Module), and their outputs were fed into a two-channel multiple-tau correlator (Correlator.com). The signal-to-noise ratio was improved by cross-correlating these two channels. With respect to the primary beam, depolarized scattering was observed via crosspolarizers. The incoming laser beam passed through a GlanThompson polarizer with an extinction ratio of $10^{-6}$, and another Glan-Thompson polarizer, with an extinction ratio of $10^{-8}$, was mounted in front of the collection optics. The instrumental depolarization was controlled by measuring a suspension of Fetal Bovine Serum (FBS, Invitrogen, Switzerland) diluted in phosphate buffer (10\% Vol./Vol.).

\section{THEORY, RESULTS, AND DISCUSSION}

Optical anisotropy may arise from either shape or refractive index. High-resolution transmission electron microscopy (HRTEM) images show (Figure 2) that Au NPs display (i) internal nanostructural anisotropy, i.e., the distinct domains of atomic arrays (Figure $2 \mathrm{~B} / \mathrm{C}$ ) stemming from the polycrystalline nature typically found in Au NPs in the range above $20 \mathrm{~nm},{ }^{13,14}$

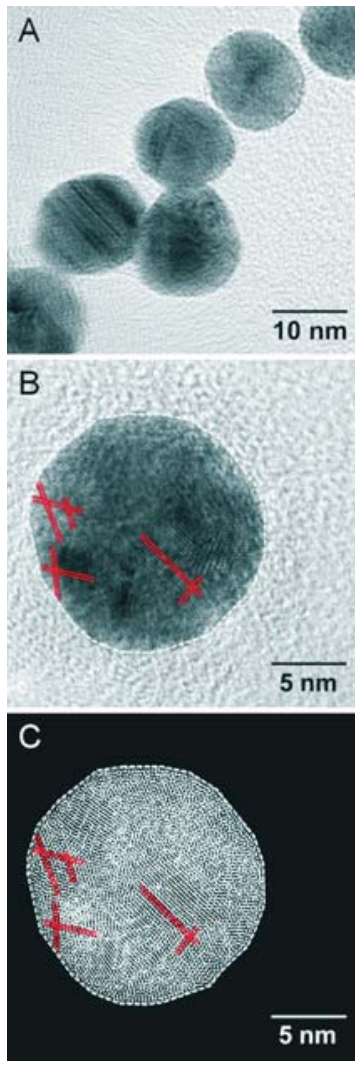

Figure 2. High-resolution TEM micrographs of Au NPs. The morphological perturbations disrupting the spherical symmetry and resulting in a small yet important shape anisotropy are apparent, and the domains of atomic arrays can easily be identified (A/B). After Fourier filtering and contrast enhancement, the internal lattice structures highlight the polycrystalline nature of these Au NPs (C). A few domains are indicated in red. The spacing between planes is $0.23 \mathrm{~nm}$, corresponding to the (111) interplanar distance of fcc structure.

and (ii) morphological perturbations breaking the spherical symmetry, due to the face-centered cubic (fcc) lattice. ${ }^{15,16}$

These inherent features of round $\mathrm{Au}$ NPs result in a small but relevant optical anisotropy affecting the Au NP properties. ${ }^{17}$ Indeed, their depolarization factor $\left(D_{\mathrm{f}} \equiv I_{\mathrm{vh}} / I_{\mathrm{vv}}\right)$, where $I_{\mathrm{vh}}$ and $I_{\mathrm{vv}}$ are the depolarized and polarized scattering intensities, respectively, is above 0.1 (Figure 3). The depolarization factor of FBS is practically zero, which confirms that there is no instrumental depolarization.

Given the optical anisotropy of the Au NPs, the temporal fluctuations in depolarized scattering can be deployed. Photon correlation spectroscopy self-correlates the time-dependent fluctuations of the intensity of the scattered laser light. ${ }^{5}$ For noninteracting uniform particles, the depolarized (vh) and polarized (vv) field autocorrelation functions are written as follows:

$$
\begin{aligned}
& \mathrm{g} 1_{\mathrm{vh}}(t)=\mathrm{e}^{-\Gamma_{\mathrm{T}} t} \mathrm{e}^{-\Gamma_{\mathrm{R}} t} \\
& \mathrm{~g} 1_{\mathrm{vv}}(t)=y \mathrm{e}^{-\Gamma_{\mathrm{T}} t}+(1-y) \mathrm{g} 1_{\mathrm{vh}}(t)
\end{aligned}
$$

where $1>y>0$. In case of spherical particles, the exponents are as follows: ${ }^{18-21}$

$$
\Gamma_{\mathrm{T}} \equiv q^{2} \frac{k_{\mathrm{B}} T}{6 \pi \eta} \frac{1}{r_{\mathrm{H}}}=q^{2} D_{\mathrm{T}}
$$




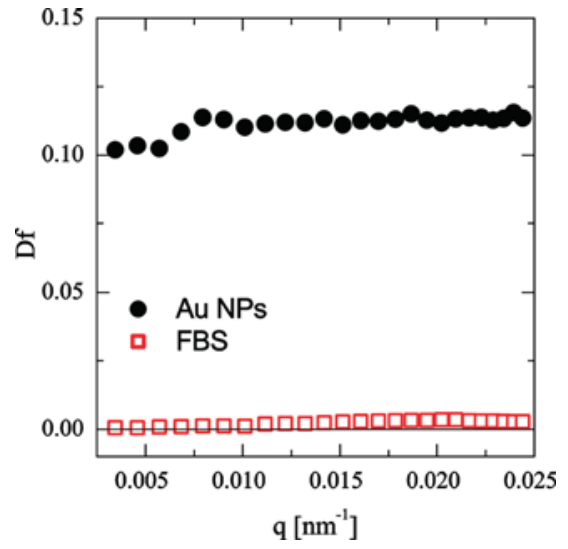

Figure 3. Depolarization factor, $D_{\mathrm{f}} \equiv I_{\mathrm{vh}} / I_{\mathrm{vv}}$ of the gold nanoparticles and fetal bovine serum shown as a function of the momentum transfer (scattering angles: $\theta=15^{\circ}-135^{\circ}$ ).

$$
\Gamma_{\mathrm{R}} \equiv \frac{3 k_{\mathrm{B}} T}{4 \pi \eta} \frac{1}{r_{\mathrm{H}}^{3}}=6 D_{\mathrm{R}}
$$

where $r_{\mathrm{H}}$ is the hydrodynamic radius, $k_{\mathrm{B}}$ the Boltzmann constant, $T$ the temperature, $\eta$ the viscosity of the solvent, $q$ the momentum transfer $q=(4 \pi / \lambda) n \sin (\theta / 2), \theta$ the scattering angle, $\lambda$ the wavelength of the scattered waves, and $n$ the refractive index of the solution. Equations 3 and 4 correspond to translational $\left(D_{\mathrm{T}}\right)$ and rotational $\left(D_{\mathrm{R}}\right)$ diffusion, respectively. Rotational diffusion is independent of the angle of observation and dominates at low angles where $q \approx 0$. The hydrodynamic radius defines the relaxation rate of both correlation functions. It is important to point out that for particles with optical anisotropy, the rotational and translational dynamics are found entangled in the field correlation functions. Therefore, if the rotational diffusion is not accounted for, the interpretation becomes misleading, e.g., by intuitively assuming aggregates or more than one population of NPs. If properly accounted for, however, this approach provides enhanced resolution for obtaining the particle size and polydispersity of small nanoparticles.

For polydisperse NPs, which are actually more frequent than uniform particles, the field correlation functions are expressed as the Laplace transform of the probability density function describing the dispersion in the relaxation rate. Accordingly, the correlation function of the depolarized scattering is written as follows:

$$
\mathrm{g} 1_{\mathrm{vh}}(t)=\int_{0}^{\infty} \mathrm{d} \Gamma \mathrm{P}_{\Gamma}(\Gamma) \mathrm{e}^{-\Gamma t}
$$

where $\Gamma=\Gamma_{\mathrm{R}}+\Gamma_{\mathrm{T}}$. Equation 5 can be expanded into a series of the central moments of the probability density function $P_{\Gamma}(\Gamma)$ (cumulant expansion): ${ }^{22}$

$$
\mathrm{g}_{\mathrm{vh}}(t)=\mathrm{e}^{-\langle\Gamma\rangle t}\left(1+\sum_{n=2}^{\infty}(-1)^{n} \frac{M_{n}}{n !} t^{n}\right)
$$

where $\langle\Gamma\rangle$ is the average rate of relaxation,

$$
\langle\Gamma\rangle \equiv \int_{0}^{\infty} \mathrm{d} \Gamma \Gamma \mathrm{P}_{\Gamma}(\Gamma)
$$

and the central moments, $M_{n}$ are defined as follows:

$$
\langle\Gamma\rangle \equiv \int_{0}^{\infty} \mathrm{d} \Gamma(\Gamma-\langle\Gamma\rangle)^{\mathrm{n}} P_{\Gamma}(\Gamma)
$$

Equation 6 is frequently written by using the natural logarithm of the correlation function:

$$
\ln g 1_{\mathrm{vh}}(t)=-\langle\Gamma\rangle t+\frac{M_{2}}{2} t^{2}+\frac{M_{3}}{6} t^{3}+\ldots
$$

and the dispersion in $\Gamma$ is quantified by the average-normalized standard deviation:

$$
\sigma(\Gamma) \equiv \sqrt{M_{2}} /\langle\Gamma\rangle
$$

Figure $4 \mathrm{~A}$ shows $\mathrm{g} 1_{\mathrm{vh}}(t)$ recorded at the lowest angle available on our instrument, as well as the best fits obtained by the cumulant expansion (eq 9). The cumulant expansion estimates that the dispersion in $\langle\Gamma\rangle$ is $\sigma(\Gamma)=49 \%$. It follows from eqs 1 , 3 , and 4 that the relationship between $\langle\Gamma\rangle=\left\langle\Gamma_{\mathrm{R}}\right\rangle+\left\langle\Gamma_{\mathrm{T}}\right\rangle$ and $q^{2}$ is described by a straight line (Figure 4B), and as a matter of fact, $\Gamma_{\mathrm{R}}$ and $\Gamma_{\mathrm{T}}$ are respectively equal to the intercept and slope of this line. Relying on the best linear fit that estimates the linear relationship between $\langle\Gamma\rangle$ and $q^{2},\left\langle r_{\mathrm{H}}\right\rangle=23 \mathrm{~nm}$ is obtained by simultaneously solving eqs 3 and 4 . The statistical moments $M_{\mathrm{n}}$ uniquely define the corresponding probability density function. The cumulant expansion can provide, in
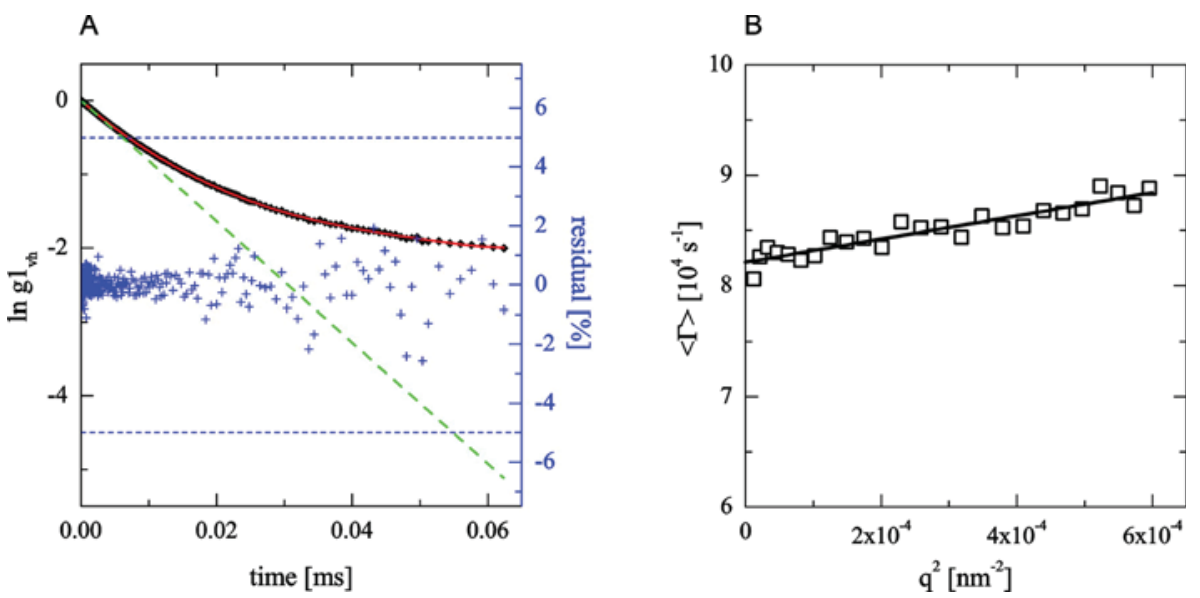

Figure 4. Panel A: Natural logarithm of the depolarized field autocorrelation function recorded at $\theta=15^{\circ}$ (full symbol), and the best fit of the cumulant expansion (solid line). The dashed straight line corresponds to the average size given by the cumulant expansion. Panel B: Relaxations of $\mathrm{g} 1_{\mathrm{vh}}$ estimated by the cumulant expansion as a function of the squared momentum transfer $(\theta=15,20, \ldots, 135)$. The solid line is the best linear fit. 
A

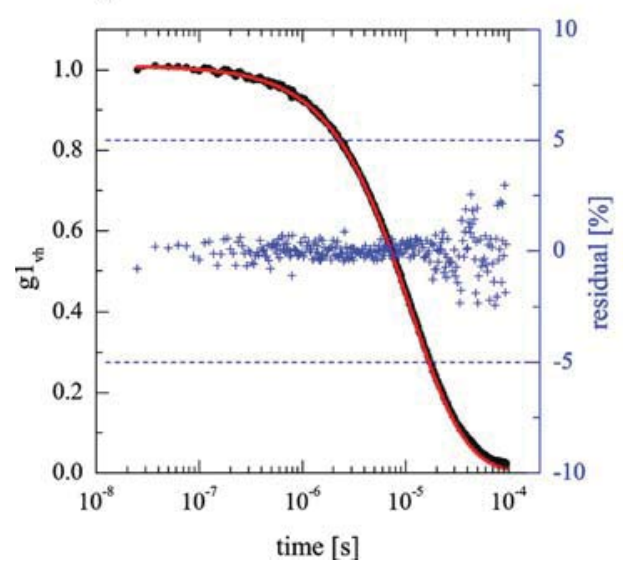

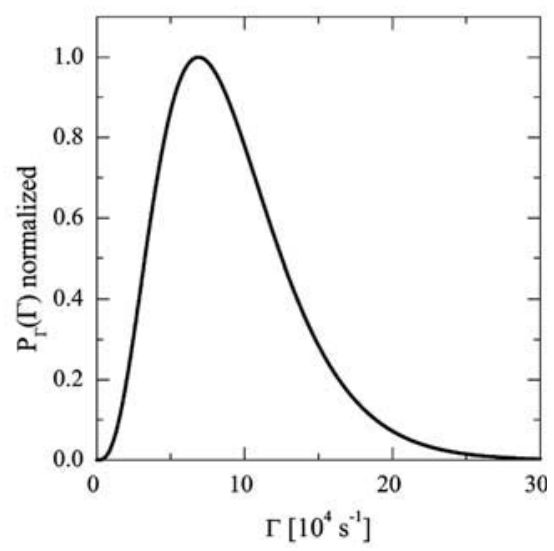

Figure 5. Panel A: Experimental depolarized field autocorrelation function recorded at $\theta=15^{\circ}$ (full symbol), the best fit of eq 13 (solid line), and the residual between the experimental data and the best fit (in blue color). Panel B: The distribution of the relaxation rates resulting in the best fit $\left(\langle\Gamma\rangle=91165 \mathrm{~s}^{-1}\right.$ and $\left.\sigma(\Gamma)=50 \%\right)$.

principle, a complete description of the intensity-weighted probability density function $P_{\Gamma}(\Gamma)$. In practice, however, it is not trivial to determine central moments of higher order as these statistical moments dominate only at long lag times, where noise usually corrupts photon correlation data. ${ }^{23}$ This noise causes a high-order cumulant fit to be highly oscillatory and nonphysical due to the polynomial character.

To circumvent the problem, another approach is taken on, and a reliable estimate for $P_{\Gamma}(\Gamma)$ is found. The motivation is to estimate directly the probability density function of the hydrodynamic radius, $P_{\mathrm{r}_{\mathrm{H}}}\left(r_{\mathrm{H}}\right)$, via the rule of transforming random variables. ${ }^{24}$ Let $\Phi$ represent the relationship between $\Gamma\left(=\Gamma_{\mathrm{T}}+\Gamma_{\mathrm{R}}\right)$ and $r_{\mathrm{H}}$ (eqs 3 and 4 ). Then the probability density function of the intensity-weighted hydrodynamic radius is given by the following:

$$
P_{r_{\mathrm{H}}}\left(r_{\mathrm{H}}\right)=P_{\Gamma}\left(\Phi\left(r_{\mathrm{H}}\right)\right) \cdot\left|\frac{\mathrm{d}}{\mathrm{d} r_{\mathrm{H}}} \Phi\left(r_{\mathrm{H}}\right)\right|
$$

Given that these Au NPs are considerably smaller than the wavelength of the laser, the scattered intensity is proportional to the square of the particle volume. ${ }^{25}$ Therefore, the numberweighted probability density function can be obtained from the intensity-weighted probability density function by normalizing with the square of the particle volume and form factor:

$$
P_{r_{\mathrm{H}}}^{*}=\frac{P_{r_{\mathrm{H}}}}{\mathrm{V}^{2} \cdot \phi} / \int_{0}^{\infty} \mathrm{d} r_{\mathrm{H}} \frac{P_{r_{\mathrm{H}}}}{\mathrm{V}^{2} \cdot \phi}
$$

where $V(r)=(4 / 3) \pi r^{3}$ is the volume and $\phi(q, r)=\{(3 /$ $\left.\left.(q r)^{3}\right)[\sin (q r)-q r \cos (q r)]\right\}^{2}$ is the form factor of a spherical particle of radius $r$. Equation 12 simplifies if we take into account that on the available $q$-range of the light scattering instrument, $\phi(q, r)$ of such small NPs is practically one as $q r \ll$ 1. Relying on TEM, it can be excluded that the Au NPs are multimodal, and $P_{\Gamma}(\Gamma)$ is modeled by the Schulz-Zimm distribution: ${ }^{26,27}$

$$
P_{\Gamma}(\Gamma)=\frac{1}{\operatorname{Gamma}\left(\frac{1}{\sigma^{2}}\right)} \mathrm{e}^{-\Gamma /\langle\Gamma\rangle \sigma^{2}}\left(\frac{1}{\langle\Gamma\rangle \sigma^{2}}\right)^{1 / \sigma^{2}} \Gamma^{1 / \sigma^{2}-1}
$$

The parameters $\langle\Gamma\rangle$ and $\sigma$ are defined in eqs 7 and 10, and in case of unimodal distribution $0<\sigma \leq 1$. When $\sigma$ is close to 1 , the Schulz-Zimm distribution approaches an exponential distribution, and when $\sigma$ is small, it approaches a Gaussian distribution. According to eq 5 , the depolarized correlation function is now written as follows:

$$
\mathrm{g} 1_{\mathrm{vh}}(t)=\left(1+\langle\Gamma\rangle \sigma^{2} t\right)^{-1 / \sigma^{2}}(0 \leq \sigma \leq 1)
$$

Figure $5 \mathrm{~A}$ shows $\mathrm{g} 1_{\mathrm{vh}}(t)$ recorded at $\theta=15^{\circ}$, the best fit of the expression given by eq 14 , and the residual between data and best fit. The corresponding Schulz-Zimm distribution is highlighted in Figure 5B.

The number-weighted probability density function of the hydrodynamic radius is estimated via eqs 11 and 12 , and the result in comparison with the TEM results is shown in Figure 6.
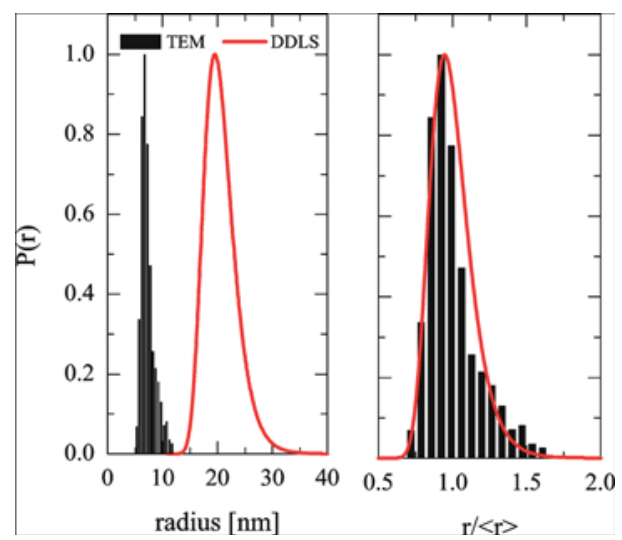

Figure 6. Au NP size distributions obtained by TEM and DDLS. Data are normalized by the amplitude. Left: The TEM histogram is built from counting and classifying $1775 \mathrm{Au}$ NPs (average $=7.5 \mathrm{~nm}$ and SD $=1.3 \mathrm{~nm}$ ). The number-weighted distribution of the hydrodynamic radius is obtained via eqs 11 and 12, using the results shown in Figure $5 \mathrm{~B}$. Right: the distributions are plotted around their respective average value.

The number-average hydrodynamic radius is estimated by the following:

$$
\left\langle r_{\mathrm{H}}\right\rangle=\int_{0}^{\infty} \mathrm{d} r_{\mathrm{H}} r_{\mathrm{H}} P_{r_{\mathrm{H}}}^{*}\left(r_{\mathrm{H}}\right)
$$

and the polydispersity by the following: 

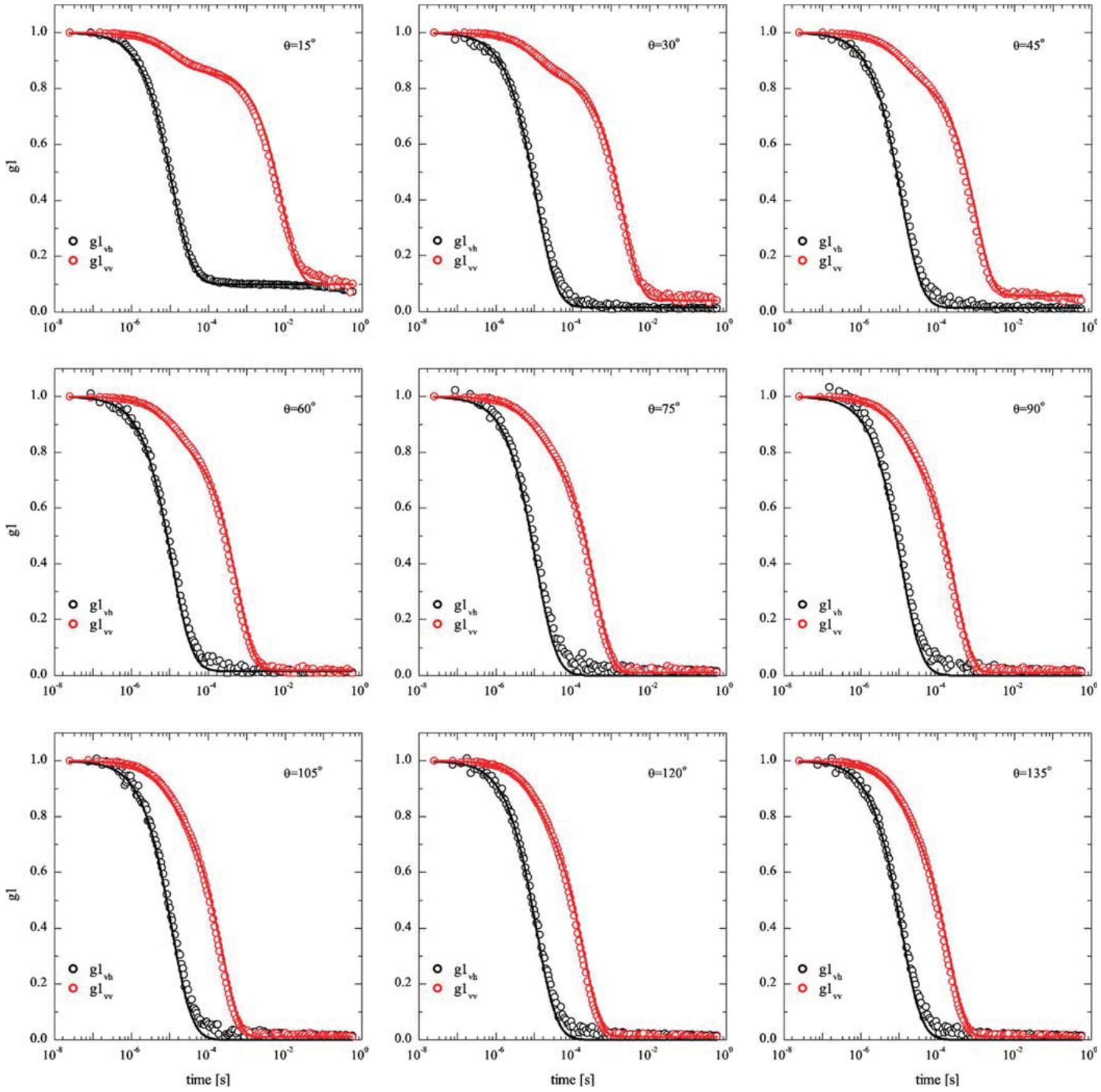

Figure 7. Experimental (symbol) and reconstructed (solid line) depolarized (vh) and polarized (vv) autocorrelation functions $\left(\theta=15^{\circ}-135^{\circ}\right.$ ). The reconstructed correlation functions are obtained via eqs 11,17 , and 18, with only one adjustable parameter: $y(0.85>y>0.82)$. We used $P_{r_{\mathrm{H}}}\left(r_{\mathrm{H}}\right)$ that was obtained at $\theta=15^{\circ}$.

$$
\sigma\left(r_{\mathrm{H}}\right)=\frac{1}{\left\langle r_{\mathrm{H}}\right\rangle} \sqrt{\int_{0}^{\infty} \mathrm{d} r_{\mathrm{H}}\left(r_{\mathrm{H}}-\left\langle r_{\mathrm{H}}\right\rangle\right)^{2} P_{r_{\mathrm{H}}}^{*}\left(r_{\mathrm{H}}\right)}
$$

resulting in $\left\langle r_{\mathrm{H}}\right\rangle=21 \mathrm{~nm}$ and $\sigma\left(r_{\mathrm{H}}\right)=15 \%$

The two distributions have the same shape, indicating that the main source of polydispersity arises from the size distribution of the Au NPs and that the polymer shell does not increase the dispersion in particle size. This becomes obvious when plotting both distributions around their respective average value (Figure 6 , right), where we find a very good agreement between TEM and DDLS. The difference between the average core radius of $\left\langle r_{\mathrm{TEM}}\right\rangle$ and $\left\langle r_{\mathrm{H}}\right\rangle$ (Figure 6,

left) thus provides us with an estimate of the polymer shell thickness around the particles. The resulting value of $\left\langle r_{\mathrm{H}}\right\rangle-$ $\left\langle r_{\mathrm{TEM}}\right\rangle=13.5 \mathrm{~nm}$ can be compared with an estimate of the contour length and the radius of gyration of the free polymer chain as the upper and lower limits of the shell thickness. Here we consider that the molar mass of the PEG monomer unit, $M$, is between 44 and $45 \mathrm{~g} / \mathrm{mol}$, and thus, the average number of monomer units is $M_{\mathrm{w}} / M=111-114$. The length of a $\mathrm{C}-\mathrm{C}$ bond is $0.154 \mathrm{~nm}$, and that of a $\mathrm{C}-\mathrm{O}$ bond is $0.143 \mathrm{~nm}$. Taking into account the bond angles, we estimate that the length of the PEG monomer is $0.316 \mathrm{~nm}$, the contour length of the polymer of $\mathrm{Mw}=5000 \mathrm{~g} / \mathrm{mol}$ is approximately $35-36 \mathrm{~nm}$, 


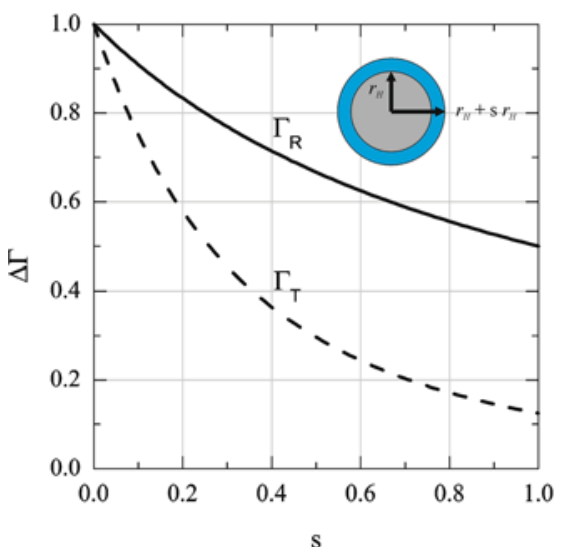

B

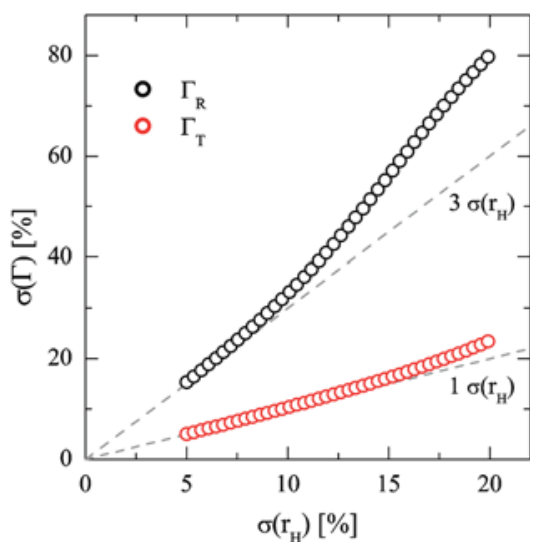

Figure 8. Panel A: Comparison of the relative changes in rotational and translational relaxation rates as a function of the relative changes in NP size $\Delta \Gamma \equiv \Gamma\left(r_{\mathrm{H}}\right) / \Gamma\left(r_{\mathrm{H}}+s r_{\mathrm{H}}\right)$. Panel B: Dispersion in the relaxation rates describing rotational and translational diffusion (eqs $\left.1-4\right)$ as a function of the dispersion of the hydrodynamic radius in the range of $5 \% \leq \sigma\left(r_{\mathrm{H}}\right) \leq 20 \%$. Dispersion is defined as the mean-normalized standard deviation $\sigma(x)=$ $\mathrm{SD} /\langle x\rangle$. The dispersion of the translational relaxation rates $\sigma\left(\Gamma_{\mathrm{T}}\right)$ is nearly equal to the dispersion of the hydrodynamic radius, while the dispersion of the rotational relaxation rates $\sigma\left(\Gamma_{\mathrm{R}}\right)$ is at least three times higher when $\sigma\left(r_{\mathrm{H}}\right)>10 \%$.

whereas the average radius of gyration $\left\langle R_{\mathrm{G}}\right\rangle$ would be $\sim 3.3 \mathrm{~nm}$ assuming a random-coil conformation. The experimentally determined shell thickness of $13.5 \mathrm{~nm}$ is thus indeed between these two limiting values, in agreement with what is expected from the grafting density of $1.2-2$ PEG chains $/ \mathrm{nm}^{2}{ }^{28,29}$ It is also consistent with earlier reports that PEG chains partially adsorb onto the Au surface via hydrogen bonding with the citrate ions, which results in a bending conformation with a thicker polymer layer than that of $\left\langle R_{\mathrm{G}}\right\rangle$ of the individual polymer in solution. ${ }^{28-30}$

The consistency of our approach with the experimental data is tested by reconstructing the polarized and depolarized correlation functions based on $P_{\mathrm{r}_{\mathrm{H}}}\left(r_{\mathrm{H}}\right)$ (eq 11):

$$
\begin{aligned}
& \mathrm{g} 1_{\mathrm{vh}}^{*}(t)=\int_{0}^{\infty} \mathrm{d} r_{\mathrm{H}} P_{r_{\mathrm{H}}}\left(r_{\mathrm{H}}\right) \mathrm{e}^{-\left(q^{2} \frac{k_{\mathrm{B}} T}{6 \pi \eta r_{\mathrm{H}}}+\frac{6 k_{\mathrm{B}} T}{4 \pi \eta r_{\mathrm{H}}^{3}}\right) t} \\
& \mathrm{~g} 1_{\mathrm{vv}}^{*}(t)=y \int_{0}^{\infty} \mathrm{d} r_{\mathrm{H}} P_{r_{\mathrm{H}}}\left(r_{\mathrm{H}}\right) \mathrm{e}^{-q^{2} \frac{k_{\mathrm{B}} T 1}{6 \pi r_{\mathrm{H}}} t}+(1-y) \mathrm{g} 1_{\mathrm{vh}}^{*}(t)
\end{aligned}
$$

which are then weighted against the experimental data recorded at different angles (Figure 7). $P_{r_{\mathrm{H}}}\left(r_{\mathrm{H}}\right)$ was obtained at $\theta=15^{\circ}$, and the only free parameter to be adjusted is $y$, which is angle and particle-type dependent. ${ }^{4,31}$ In the case of our Au NPs, values between 0.85 and 0.82 result in an overall excellent agreement.

It follows from eqs 3 and 4 that compared to translational relaxation, rotational relaxation is more sensitive to relative changes in morphology or size (Figure 8A), which enables a highly sensitive in situ characterization of, among others, NP surface functionalization or interaction with biological matrixes. This is a field of high interest, ${ }^{32}$ for as soon as NPs enter the body and bloodstream, they find a complex environment populated by bio- and small molecules such as proteins, salts, vitamins, and lipids. To understand and control the behavior and fate of NPs in such settings is fundamental for harnessing their potentially beneficial properties and for mitigating the risks they may pose to human health and the environment. ${ }^{33}$ Additionally, when NPs are polydisperse, the rotational and translational diffusion coefficients exhibit dispersion. Interest- ingly, while the number-weighted size polydispersity of the NPs is rather moderate (i.e., $\sigma\left(r_{\mathrm{H}}\right)=15 \%$ ) the polydispersity of $\Gamma$, being dominated by $\Gamma_{\mathrm{R}}$ at low scattering angles, is considerably larger (Figure $5 \mathrm{~B}$ ). This enhancement in the dispersion width is due to the increased sensitivity of the relaxation rate as a function of $r_{\mathrm{H}}: \Gamma \propto 1 / r_{\mathrm{H}}^{3}$ (Figure 8A). To demonstrate this amplification in the dispersion, $\Gamma_{\mathrm{R}}$ and $\Gamma_{\mathrm{T}}$ were calculated as a function of dispersion in $r_{H}$ in the case of normally distributed $\mathrm{r}_{\mathrm{H}}$ values. Dispersion is defined as the mean-normalized standard deviation: $\sigma(x)=\mathrm{SD} /\langle x\rangle$. Figure $8 \mathrm{~B}$ shows that rotational relaxation has another advantage over translation relaxation, for it is extremely sensitive for detecting and resolving even small polydispersities.

\section{CONCLUSIONS}

Small round metallic nanoparticles promote depolarized dynamic light scattering, owing to inherent properties such as localized surface plasmon resonances and intrinsic optical anisotropy. The high sensitivity of the relaxation of the depolarized scattering to rotational diffusion enables an accurate estimate of polydispersity and size. Given the wide range of applications of Au NPs in complex environments, this approach offers new opportunities for an otherwise often challenging characterization, through simple, noninvasive and in situ depolarized dynamic light scattering experiments.

\section{AUTHOR INFORMATION}

\section{Corresponding Author}

*Phone: +41 26300 8964; e-mail: sandor.balog@unifr.ch.

\section{Author Contributions}

This manuscript was written through contributions of all authors. All authors have given approval to the final version of the manuscript.

\section{Author Contributions}

"These authors contributed equally.

\section{Notes}

The authors declare no competing financial interest. 


\section{ACKNOWLEDGMENTS}

S.B., L.R.-L., C.A.M., B.M., B.R.-R. and A.P.-F. are grateful for the financial support of the Adolphe Merkle Foundation. L.R.L. acknowledges the financial support from the L'Oreal Switzerland and UNESCO's fellowship program "For Women in Science 2013". The authors would like to thank Herbert Schaffer for the expertise and assistance in HRTEM. The support of the Dr. Alfred Bretscher Fund is gratefully acknowledged, and access to TEM was kindly provided by the Microscopy Imaging Centre of the University of Bern. P.S. acknowledges financial support from the Swedish Research Council VR through the Linnaeus Center of Excellence on Organizing Molecular Matter. The authors also acknowledge financial support from the European Commission under the Seventh Framework Program by means of the grant agreement for the Integrated Infrastructure Initiative No. 262348 European Soft Matter Infrastructure (ESMI).

\section{REFERENCES}

(1) Tsuzuki, T. Commercial Scale Production of Inorganic Nanoparticles. Int. J. Nanotechnol. 2009, 6, 567-578.

(2) Giljohann, D. A.; Seferos, D. S.; Daniel, W. L.; Massich, M. D.; Patel, P. C.; Mirkin, C. A. Gold Nanoparticles for Biology and Medicine. Angew. Chem.-Int. Ed. 2010, 49, 3280-3294.

(3) Kelly, K. L.; Coronado, E.; Zhao, L. L.; Schatz, G. C. The Optical Properties of Metal Nanoparticles: The Influence of Size, Shape, and Dielectric Environment. J. Phys. Chem. B 2002, 107, 668-677.

(4) Khlebtsov, N. G.; Mel'nikov, A. G.; Bogatyrev, V. A.; Alekseeva, A. V.; Khlebtsov, B. N. Depolarization of Light Scattered by Gold Nanospheres and Nanorods. Opt. Spectrosc. 2006, 100, 448-455.

(5) Pecora, R. Dynamic Light Scattering: Applications of Photon Correlation Spectroscopy; Plenum Press: New York, 1985.

(6) Haghighi, M.; Plum, M. A.; Gantzounis, G.; Butt, H.-J.; Steffen, W.; Fytas, G. Plasmon-Enhanced Dynamic Depolarized Light Scattering. J. Phys. Chem. C 2013, 117, 8411-8419.

(7) Rodríguez-Fernández, J.; Pérez-Juste, J.; Liz-Marzán, L. M.; Lang, P. R. Dynamic Light Scattering of Short Au Rods with Low Aspect Ratios. J. Phys. Chem. C 2007, 111, 5020-5025.

(8) Chen, S.; Kraus, T. Nanorod-Depolarized Dynamic Light Scattering in a Gelling Liquid. J. Phys. Chem. C 2012, 116, 1676616775.

(9) Glidden, M.; Muschol, M. Characterizing Gold Nanorods in Solution Using Depolarized Dynamic Light Scattering. J. Phys. Chem. C 2012, 116, 8128-8137.

(10) Haghighi, M.; Tahir, M. N.; Tremel, W.; Butt, H. J.; Steffen, W. Translational and Rotational Diffusion of Gold Nanorods Near a Wall. J. Chem. Phys. 2013, 139, 064710-064717.

(11) Haiss, W.; Thanh, N. T. K.; Aveyard, J.; Fernig, D. G. Determination of Size and Concentration of Gold Nanoparticles from UV-Vis Spectra. Anal. Chem. 2007, 79, 4215-4221.

(12) Turkevich, J.; Stevenson, P. C.; Hillier, J. A Study of the Nucleation and Growth Processes in the Synthesis of Colloidal Gold. Discuss. Faraday Soc. 1951, 11, 55-75.

(13) Zhou, C.; Yu, J.; Qin, Y.; Zheng, J. Grain Size Effects in Polycrystalline Gold Nanoparticles. Nanoscale 2012, 4, 4228-4233.

(14) Scott, M. C.; Chen, C. C.; Mecklenburg, M.; Zhu, C.; Xu, R.; Ercius, P.; Dahmen, U.; Regan, B. C.; Miao, J. W. Electron Tomography at 2.4-Angstrom Resolution. Nature 2012, 483, 444-447.

(15) Barnard, A. S.; Young, N. P.; Kirkland, A. I.; van Huis, M. A.; $\mathrm{Xu}, \mathrm{H}$. Nanogold: A Quantitative Phase Map. ACS Nano 2009, 3, 1431-1436.

(16) Petkov, V.; Peng, Y.; Williams, G.; Huang, B.; Tomalia, D.; Ren, Y. Structure of Gold Nanoparticles Suspended in Water Studied by XRay Diffraction and Computer Simulations. Phys. Rev. B 2005, 72, 195402-195408.

(17) Watanabe-Tamaki, R.; Ishikawa, A.; Tanaka, T. Blue Shift of Plasmonic Resonance Induced by Nanometer Scale Anisotropy of
Chemically Synthesized Gold Nanospheres. Appl. Phys. Lett. 2013, 102, 043110-043114.

(18) Einstein, A. The Motion of Elements Suspended in Static Liquids as Claimed in the Molecular Kinetic Theory of Heat. Ann. Phys.-Berlin 1905, 17, 549-560.

(19) Einstein, A. Eine neue Bestimmung der Moleküldimensionen. Ann. Phys.-Berlin 1906, 324, 289-306.

(20) Einstein, A. Berichtigung zu meiner Arbeit: Eine neue Bestimmung der Moleküldimensionen. Ann. Phys-Berlin 1911, 339, 591-592.

(21) Debye, P. Polar Molecules; Dover: New York, 1929.

(22) Koppel, D. E. Analysis of Macromolecular Polydispersity in Intensity Correlation Spectroscopy: The Method of Cumulants. J. Chem. Phys. 1972, 57, 4814-4820.

(23) Schatzel, K. Noise on Photon Correlation Data. I. Autocorrelation Functions. Quant. Opt.: J. Euro. Opt. Soc. P B 1990, 2, 287-305.

(24) Peyton Z. Peebles, J. Probability, Random Variables, and Random Signal Principles; McGraw-Hill: New York, 1987.

(25) Bohren, C. F. Absorption and Scattering of Light by Small Particles. Wiley: New York, 1983.

(26) Schulz, G. V. The Kinetics of Chain Polymerisation V. The Influence of Various Types of Reactions on the Poly-molecularity. $Z$. Phys. Chem. B-Chem. E 1939, 43, 25-46.

(27) Zimm, B. H. Apparatus and Methods for Measurement and Interpretation of the Angular Variation of Light Scattering: Preliminary Results on Polystyrene Solutions. J. Chem. Phys. 1948, 16, 1099-1116.

(28) Larson-Smith, K.; Pozzo, D. C. Competitive Adsorption of Thiolated Poly(ethylene glycol) and Alkane-Thiols on Gold Nanoparticles and Its Effect on Cluster Formation. Langmuir 2012, 28, 13157-13165.

(29) Walkey, C. D.; Olsen, J. B.; Guo, H. B.; Emili, A.; Chan, W. C. W. Nanoparticle Size and Surface Chemistry Determine Serum Protein Adsorption and Macrophage Uptake. J. Am. Chem. Soc. 2012, 134, 2139-2147.

(30) Sekiguchi, S.; Niikura, K.; Matsuo, Y.; Ijiro, K. Hydrophilic Gold Nanoparticles Adaptable for Hydrophobic Solvents. Langmuir 2012, 28, 5503-5507.

(31) Khlebtsov, N. G.; Melnikov, A. G.; Bogatyrev, V. A.; Dykman, L. A.; Alekseeva, A. V.; Trachuk, L. A.; Khlebtsov, B. N. Can the Light Scattering Depolarization Ratio of Small Particles Be Greater Than 1/ 3? J. Phys. Chem. B 2005, 109, 13578-13584.

(32) Lynch, I.; Cedervall, T.; Lundqvist, M.; Cabaleiro-Lago, C.; Linse, S.; Dawson, K. A. The Nanoparticle - Protein Complex as a Biological Entity: A Complex Fluids and Surface Science Challenge for the 21st Century. Adv. Colloid Interface Sci. 2007, 134-35, 167-174.

(33) Bouwmeester, H.; et al. Minimal Analytical Characterization of Engineered Nanomaterials Needed for Hazard Assessment in Biological Matrices. Nanotoxicology 2010, 5, 1-11. 